\title{
Comentarios sobre "Capacidad predictiva de los parámetros hemodinámicos no invasivos para hipotensión durante cesárea: un estudio prospectivo observacional"
}

\author{
Comments on "The predictive ability of non-invasive haemo- \\ dynamic parameters for hypotension during caesarean sec- \\ tion: a prospective observational study"
}

Yokose M. y cols.: The predictive ability of non-invasive haemodynamic parameters for hypotension during caesarean section: a prospective observational study. Anaesthesia 2015; 70: 555-62

JUAN FORERO G. ${ }^{1}$, EDUARDO KATTAN ${ }^{1}$, HÉCTOR J. LACASSIE²

\begin{abstract}
Introduction: A review of the Yokose et al article was performed. The purpose of this study was to determine whether non-invasive parameters derived from pulse oximeter measurements could predict hypotension after administering spinal anesthesia for cesarean section. Hypotension can trigger maternal nausea/vomiting and/or impairment of the utero-placental perfusion and the fetal well-being. Therefore it is important to predict and prevent this event. Objective: To perform a critical analysis of the article with emphasis in the internal and external validity of the results and conclusions. Materials and Methods: 95 cesarean section patients were studied. Before anesthesia, non invasive hemodynamic parameters were measured: perfusion index, pleth variability index, heart rate, ratio of low-frequency to high-frequency variability index, entropy of heart rate variability, as well as non invasive blood pressure (NIBP). Surgery started after achieving a sixth thoracic segment (T6) sensory block level with a combined spinal-epidural anesthesia. A colloid coload was administered. NIBP was measured every minute. Hypotension was defined as a systolic arterial presssure less than $80 \mathrm{mmHg}$ or the appearence of clinical symptomatology. The hypotensive episode duration was not quantified. Results and Conclusions: This is a prospective, observational study demonstrating that the only predictor for arterial hypotension is heart rate, albeit with moderate sensibility and poor spe-
\end{abstract}

Key words: Cesarean, spinal anesthesia, hypotension, monitoring, physiologic, forecasting.

\footnotetext{
División de Anestesiología, Facultad de Medicina, Pontificia Universidad Católica de Chile.

Residente de Anestesiología.

2 Profesor Asociado en Anestesiología.

Correspondencia:
}

Héctor J. Lacassie

Marcoleta 377, $4^{\circ}$ piso, Deparatamento de Anestesiología, Hospital Clínico Pontificia Universidad Católica de Chile, Santiago, Chile.

lacassie@med.puc.cl 
cificity. Groups were comparable. Pre anesthesia heart rate was significantly higher in the hypotension group $\left(84 \pm 10\right.$ versus $77 \pm 13$ [beats $\bullet \mathrm{min}^{-1}$ ]; $p=$ $0,008)$.

\section{RESUMEN}

Introducción: Se realizó una revisión del estudio de Yokose y cols., quienes investigaron si los parámetros hemodinámicos no invasivos, derivados de la señal del oxímetro de pulso, podían predecir la aparición de hipotensión arterial post anestesia espinal para operación cesárea. La hipotensión puede comprometer la perfusión útero placentaria, además de generar sintomatología materna, por lo que es importante predecirla y prevenirla. Objetivo: Realizar un análisis crítico del estudio en cuanto a la validez interna y externa de los resultados y conclusiones. Materiales y Métodos: Se estudiaron 95 pacientes embarazadas que se someterían a cesárea electiva. Previo a la anestesia se midieron parámetros hemodinámicos no invasivos: índice de perfusión, índice de variabilidad pletismográfico, frecuencia cardíaca, variabilidad de la frecuencia cardíaca y entropía, además de presión arterial (PA) no invasiva. Se realizó una anestesia combinada espinal-epidural y la incisión quirúrgica se efectuó cuando el bloqueo sensitivo alcanzó a T6. Se cohidrató con coloides y se midió PA cada minuto. Se definió como hipotensión arterial a toda cifra de PA sistólica (PAS) menor de $80 \mathrm{mmHg}$ o sintomatología clínica sin cuantificar duración del episodio hipotensivo. Resultados y Conclusión: Se analizó un estudio prospectivo y observacional. LoS grupos fueron comparables. La frecuencia cardíaca (FC) pre-anestesia fue significativamente mayor en el grupo hipotensión ( $84 \pm 10$ versus $77 \pm 13$ [lat $\bullet \mathrm{min}^{-1}{ }^{-1}$; $p=0,008$ ). No hubo diferencias clínicas ni estadísticas de los otros parámetros medidos. La frecuencia cardíaca fue el único predictor de hipotensión arterial, con moderada sensibilidad y nula especificidad.
Palabras clave: Cesárea, anestesia espinal, hipotensión, monitoreo fisiológico, predicción.

\section{Introducción}

— ste trabajo presenta un análisis crítico del artícuo de Yokose y cols' ${ }^{1}$.

La operación cesárea se realiza habitualmente bajo anestesia espinal. Un efecto indeseable de ésta es la hipotensión arterial, la cual puede tener consecuencias nefastas tanto en la madre como en el feto por compromiso de la perfusión útero-placentaria. La posibilidad de predecir hipotensión arterial después de la anestesia espinal pudiera ofrecer una oportunidad de tomar medidas preventivas.

El tono vasomotor periférico basal ${ }^{2}$, la volemia ${ }^{3}$ y la actividad simpática ${ }^{4}$, influyen en el grado de hipotensión post anestesia espinal, por lo tanto, se planteó buscar métodos no invasivos que pudieran predecirla. Se utilizaron parámetros hemodinámicos derivados de las señales del oxímetro de pulso y electrocardiograma como: 1) Índice de perfusión (IP): refleja el tono vasomotor al medir la proporción entre el flujo arterial pulsátil y no pulsátil a través del lecho capilar periférico; 2) Índice de variabilidad pletismo- gráfica (IVP): traduce la variación dinámica en el IP durante el ciclo respiratorio (a mayor IVP, mayor probabilidad de respuesta a la administración de fluidos $^{5}$; 3) Frecuencia Cardíaca (FC): refleja la actividad simpática; 4) Variabilidad de la frecuencia cardíaca (VFC): estima la actividad del sistema nervioso autónomo al comparar el componente de baja frecuencia (BF), que indicada la actividad simpática y parasimpática, con respecto al componente de alta frecuencia (AF), que refleja la actividad parasimpática (una relación BF/AF alta refleja el predominio de la actividad simpática sobre la parasimpática); 5) Entropía: análisis no lineal de la VFC que traduce la estructura global de los sistemas regulatorios de la FC.

\section{Objetivo del estudio}

Determinar si los métodos de monitorización hemodinámica no invasivos usados en pacientes que se someterán a cesárea bajo anestesia espinal son efectivos para predecir hipotensión arterial. 


\section{Diseño del estudio}

Se realizó un estudio prospectivo, observacional, aprobado por el Comité de Ética institucional. Se reclutaron 95 pacientes embarazadas que se someterían a cesárea electiva en el Hospital de la Universidad de Yokohama.

Se analizan varios métodos no invasivos de medición de variables hemodinámicas para predecir hipotensión arterial en pacientes que se someten a cesárea bajo anestesia espinal. El desenlace primario buscado fue predicción de ocurrencia de hipotensión arterial (PAS $<80 \mathrm{mmHg}$ ). Se determinó el valor de corte con mejor sensibilidad y especificidad de predicción, en términos de probabilidad de ocurrencia (likelihood ratios) ${ }^{6}$. No se calculó tamaño muestral.

A través de una metodología estandarizada se evaluó el método de investigación y las conclusiones del artículo, de acuerdo a lo sugerido por Vera y cols?

\section{Resultados}

De 95 pacientes enroladas 81 terminaron el estudio (de las 14 excluidas 5 no fueron estudiados por no cumplir con el protocolo y 9 por fracasar al alcanzar el nivel de bloqueo sensorial de T6). Los grupos fueron comparables en variables antropométricas, obstétricas, neonatológicas y quirúrgicas. Cincuenta y uno presentaron hipotensión arterial (63\%).

Como el desenlace buscado es una variable categórica binaria (hipotensión: sí o no), se utilizó regresión logística para su análisis. Este identificó a la FC como el único factor independiente para predecir hipotensión arterial (OR 1,06; [IC 95\%: 1,01 -1,13]; p $=0,032$ ). Esto significa que por cada latido sobre un punto de corte (cut-off) determinado, la probabilidad de hipotensión aumenta en un $6 \%$. El modelo final sólo incluyó FC ( $p=0,012)$.

El punto de corte calculado de FC preoperatoria donde se maximiza la sensibilidad y especificidad para predecir hipotensión arterial fue de 73 lat• $\mathrm{min}^{-1}$ (sensibilidad de $86,3 \%$ (IC 95\%: 76,5 a 94,1\%) y especificidad de 50\% (IC 95\%: Cl 33,3 a 66,7\%)). No se realizó curva ROC para determinar la capacidad discriminativa del modelo.

\section{Comentarios de los revisores}

La anestesia neuroaxial en obstetricia es la técnica de elección para operación cesárea por presentar una menor incidencia de complicaciones maternas y/o fetales versus la anestesia genera|2,3. Debido a la mayor sensibilidad a los anestésicos locales durante el embarazo, la incidencia y gravedad de la hipotensión es mayor que en pacientes no gestantes ${ }^{2}$, siendo la complicación más frecuente tras una anestesia espinal ${ }^{3,4}$ con incidencias de hasta $80 \%{ }^{8}$.

Por lo tanto, prevenir la hipotensión arterial materna es uno de los objetivos anestésicos principales durante una operación cesárea. La dificulta radica en como predecirla.

La calidad de diseño del presente estudio es moderada, al no contar con un cálculo del tamaño muestral que permita cuantificar la capacidad del estudio para detectar un verdadero efecto, (poder estadístico), sin que además exista un explicación de la razón de esta omisión. Si bien en este estudio la FC pre intervención demostró capacidad predictiva de hipotensión arterial materna, debemos ser críticos de la utilidad de sus resultados.

La FC demostró ser un modesto predictor de hipotensión arterial. Sería interesante haber conocido el resultado del área bajo la curva ROC para determinar fehacientemente su capacidad predictiva.

Una sensibilidad sobre $80 \%$ es aceptable para un examen de tamizaje (screening), ya que permite detectar más casos, a costa de detectar más falsos positivos (en este caso, pacientes con FC alta que finalmente no se hipotensan post espinal). La especificidad de $50 \%$ no discrimina en absoluto ya que la capacidad de detectar los casos que no se hipotensarán no es mejor que el azar. Por otra parte, el valor predictivo positivo de $75 \%$ y negativo de $68 \%$ son cifras poco relevantes clínicamente que no cambian conducta. Los likelihood ratio positivo y negativo fueron de 1,72 y 0,28 respectivamente, por lo que la FC es una prueba muy débil para lograr cambios de conducta clínica, por ejemplo, si utilizar o no algún vasopresor de forma profiláctica (Tabla 1) 9 . Finalmente, de los otros parámetros estudiados, ninguno fue capaz de predecir hipotensión arterial. No es claro si esto fue secundario a un error tipo II, al no incluir un número adecuado de pacientes a ser estudiadas.

Analizando la validez externa o aplicabilidad de los resultados ${ }^{10}$, podemos decir que si bien la FC es un parámetro que debe estar presente en cada acto anestésico, los parámetros secundarios derivados (entropía, IP, IVP, VFC) requieren de monitores con tecnología sofisticada de mayor costo. Así, aunque fueran muy efectivos en predecir hipotensión arterial, la posibilidad actual de monitorizarlos es muy limitada, comprometiendo su aplicabilidad clínica. Por otra 


\section{Tabla 1. Resumen de las propiedades de un Test Diagnóstico}

\begin{tabular}{|c|c|c|c|}
\hline Propiedad & Descripción & Comentario & Valores de corte \\
\hline $\begin{array}{l}\text { Sensibili- } \\
\text { dad }\end{array}$ & $\begin{array}{l}\text { Capacidad para detectar a los en- } \\
\text { fermos (tabla 2) }\end{array}$ & $\begin{array}{l}\text { verdaderos (+)/enfermos. } \\
\text { Independiente de la población/prevalencia. } \\
\text { Si es muy sensible, un resultado (-) descar- } \\
\text { ta la enfermedad }\end{array}$ & Rango: $50-100 \%$ \\
\hline $\begin{array}{l}\text { Especifici- } \\
\text { dad }\end{array}$ & $\begin{array}{l}\text { Capacidad para detectar a los sanos } \\
\text { (tabla 2) }\end{array}$ & $\begin{array}{l}\text { verdaderos (-)/sanos. } \\
\text { Independiente de la población/prevalencia. } \\
\text { Si es muy específico, un resultado (+) con- } \\
\text { firma la enfermedad }\end{array}$ & Rango: $50-100 \%$ \\
\hline VPP & $\begin{array}{l}\text { Qué tan probable es que SI tenga } \\
\text { la condición si el test es (+) (tabla } 2 \text { ) }\end{array}$ & $\begin{array}{l}\text { verdaderos }(+) / \text { tests }(+) \\
\text { Depende de la población/prevalencia }\end{array}$ & Rango: $50-100 \%$ \\
\hline VPN & $\begin{array}{l}\text { Qué tan probable es que NO tenga } \\
\text { la condición si el test es (-) (tabla 2) }\end{array}$ & $\begin{array}{l}\text { verdaderos (-)/tests (-). } \\
\text { Depende de la población/prevalencia }\end{array}$ & Rango: $50-100 \%$ \\
\hline $\operatorname{LR}(+)$ & $\begin{array}{l}\text { Qué tanto más probable es encon- } \\
\text { trar un test }(+) \text { en alguien enfermo } \\
\text { comparado con alguien sano }\end{array}$ & Sensibilidad/(1-Especificidad) & $\begin{array}{l}\text { 1-2 muy poco cambio } \\
2-5 \text { cambios pequeños } \\
5-10 \text { cambios moderados } \\
>10 \text { cambios importantes }\end{array}$ \\
\hline $\operatorname{LR}(-)$ & $\begin{array}{l}\text { Qué tanto más probable es encon- } \\
\text { trar un test (-) en alguien enfermo } \\
\text { comparado con alguien sano }\end{array}$ & (1-Sensibilidad)/Especificidad & $\begin{array}{l}\text { 1-0,5 muy poco cambio } \\
0,5-0,2 \text { cambios pequeños } \\
0,2-0,1 \text { cambios moderados } \\
<0,1 \text { cambios importantes }\end{array}$ \\
\hline
\end{tabular}

VPP: predictivo positivo; VPN: valor predictivo negativo; LR: likelihood ratio; LR (+): likelihood ratio positivo; LR (-): likelihood ratio negativo. Fuente: Modificada de Salech et al (9) y Vera et al (7).

\section{Tabla 2. Matriz de un test diagnóstico}

\begin{tabular}{lccc}
\hline Test & Enfermo & Sano & Total \\
Positivo & $a$ & $b$ & $a+b$ \\
Negativo & $c$ & $d$ & $c+d$ \\
Total & $a+c$ & $b+d$ & $a+b+c+d$ \\
\hline
\end{tabular}

$\mathrm{a}=$ verdaderos positivos; $\mathrm{b}=$ falsos positivos o error tipo 1 o alfa; $\mathrm{c}=$ falsos negativos o error tipo $\mathrm{ll}$ o beta; $\mathrm{d}=$ verdaderos negativos; Enfermos totales $=(a+c)$; Sanos totales $=(b+d)$; Total Test $(+)=(a+b)$; Total Test $(-)=(c+d)$.

Es importante tener pocos falsos (+) cuando se trata de un test diagnóstico, mientras que es importante tener pocos falsos

(-) en un test de tamizaje (screening).

$$
\text { Sensibilidad }=\frac{\mathrm{a}}{\mathrm{a}+\mathrm{c}}
$$

Sensibilidad es la capacidad del test para detectar enfermedad

$$
\text { Especificidad }=\frac{\mathrm{d}}{\mathrm{b}+\mathrm{d}}
$$

Especificidad es la capacidad del test para detectar a los sanos

$$
\text { Valor predictivo Positivo }(V P P)=\frac{\mathrm{a}}{\mathrm{a}+\mathrm{b}}
$$

VPP depende del valor pre-test, es decir, de la prevalencia de la enfermedad de la población estudiada

$$
\text { Valor predictivo Negativo }(V P P)=\frac{\mathrm{d}}{\mathrm{c}+\mathrm{d}}
$$

VPN depende del valor pre-test, es decir, de la prevalencia de la enfermedad de la población estudiada 
parte, el protocolo utilizado incluye cohidratación con coloides, mientras que en la gran mayoría de los centros se utilizan cristaloides con excelentes resultados.

En resumen, sus resultados indican que una FC materna más alta es el único predictor de hipotensión arterial materna durante operación cesárea bajo anestesia espinal. Usando un valor umbral de 73 lat $\bullet \mathrm{min}^{-1}$ tiene sensibilidad de $86 \%$ y especificidad de $50 \%$, generando likelihood ratios muy modestos como para determinar un cambio de conducta clínica, por lo que no recomendamos utilizarlo.

\section{Referencias}

1. Yokose M, Mihara T, Sugawara $Y$, Goto $T$. The predictive ability of non-invasive haemodynamic parameters for hypotension during caesarean section: a prospective observational study. Anaesthesia 2015 May;70(5):555-62.

2. Ngan Kee WD. Prevention of maternal hypotension after regional anaesthesia for caesarean section. Curr Opin Anaesthesiol 2010 Jun;23(3):304-9.

3. Rosen MA, Hughes SC. G. L. Regional anesthesia for labour and delivery. In: Hughes SC, Rosen $A R$, and $S S, G L$, editores. Hughes SC, Levinson G, Rosen AR Shnider and Levinson's
Anesthesia for Obstetrics, 4a Ed. Philadelphia: Lippincott Williams \& Wilkins; 2002. p. 123-48.

4. Tsen L. Anesthesia for cesarean delivery. In: Chestnut DH, Polley LS, Wong CA, LC. T, editores. Chestnut's Obstetric Anesthesia: Principles and Practice, $4 a$ Ed. Philadelphia: Mosby; 2009. p.521-73.

5. Cannesson M, Desebbe O, Rosamel P, Delannoy B, Robin J, Bastien $O$ et al. Pleth variability index to monitor the respiratory variations in the pulse oximeter plethysmographic waveform amplitude and predict fluid responsiveness in the operating theatre. Br J Anaesth 2008 Aug;101(2):200-6.

6. Coste J, Pouchot J. A grey zone for quantitative diagnostic and screening tests. Int J Epidemiol 2003 Apr;32(2):304-13.

7. Vera PG, Letelier SL, Carvajal CJ. Guía para el análisis crítico de estudios que evalúan exámenes diagnósticos. Rev Chil Obstet Ginecol 2005;70(3):196-202.

8. Datta S. Anesthesia for cesarean delivery. In: Datta S, editor. Obstetric Anesthesia Handbook, 4a Ed. Boston: Springer; 2006. p.172-210.

9. Salech F, Mery V, Larrondo F, Rada G. Estudios que evalúan un test diagnóstico: interpretando sus resultados. Rev Med Chile 2008 Sep;136(9):1203-8.

10. Rada GG, Andrade AM. Rev Med Chile 2006;134(1):115-9. 